\title{
THE VOLATILE COMPOSITION OF COMETS
}

\author{
H. A. WEAVER \\ Space Telescope Science Institute \\ 3700 San Martin Drive \\ Baltimore, MD 21218 \\ U.S.A.
}

\begin{abstract}
Comets may be our best probes of the physical and chemical conditions in the outer regions of the solar nebula during that crucial period when the planets formed. The volatile composition of cometary nuclei, in particular, can be used to decide whether comets are the product of a condensation sequence similar to that invoked to explain the compositions of the planets and asteroids, or if comets are simply agglomerations of interstellar grains which have been insignificantly modified by the events that shaped the other bodies in the solar system. Although cometary nuclei are not generally accessible to observation, observations of cometary comae can illuminate at least some of the mysteries of the nuclei provided one has a detailed knowledge of the excitation conditions in the coma and also has access to basic atomic and molecular data on the many species present in comets. This paper examines the status of our knowledge of the volatile composition of cometary nuclei and discusses how these data are obtained.
\end{abstract}

\section{Introduction}

The volatile composition of a comet is a sensitive indicator of its formation environment. In particular, the relative abundances of molecules in cometary nuclei can be used to infer the physical and chemical state of either the solar nebula or the interstellar cloud from which the nebula condensed. However, measuring the volatile inventory in comets is a demanding task that involves combining in situ spacecraft data with observations from ground-based, airborne, rocket, and Earth-orbiting observatories.

\section{Molecules in Comet Nuclei}

\subsection{THE DOMINANT VOLATILE: $\mathrm{H}_{2} \mathrm{O}$}

During the past two decades cometary observers built a strong circumstantial case for $\mathrm{H}_{2} \mathrm{O}$ 387

D. McNally (ed.), Highlights of Astronomy, Vol. 8, 387-393.

(0) 1989 by the IAU. 
ice as the dominant volatile in nuclei. However, the apparition of comet Halley provided the first opportunity to observe the $\mathrm{H}_{2} \mathrm{O}$ molecule directly. Infrared (IR) observations of Halley in December 1985 from the Kuiper Airborne Observatory (KAO) [1] revealed intense, solarpumped fluorescent emission in the $\nu_{3}$ band of $\mathrm{H}_{2} \mathrm{O}$ near $\lambda \sim 2.65 \mu \mathrm{m}$. This same band was subsequently observed in Halley post-perihelion by the IR spectrometer on Vega (the IKS experiment) [2] and from the KAO [3,4]. Measurements by the neutral mass spectrometer (NMS) on Giotto demonstrated that $\mathrm{H}_{2} \mathrm{O}$ comprised $\geq 80 \%$ of the total volatile inventory of comet Halley [5].

Water is not particularly well-suited for constraining the formation conditions of comets because its sublimation temperature is relatively high ( $\mathrm{T}_{\text {oub }} \sim 150 \mathrm{~K}$ ), and $\mathrm{H}_{2} \mathrm{O}$ is expected to be a relatively abundant molecule in the gas phase throughout much of the solar nebula. Thus, measuring the relative abundances of the more volatile trace species in cometary nuclei is required for establishing the connection between comets and interstellar grains and between comets and possible solar nebula condensates.

\subsection{MAJOR CARBON-BEARING SPECIES: $\mathrm{CO}, \mathrm{CH}_{4}, \mathrm{CO}_{2}, \mathrm{H}_{2} \mathrm{CO}$}

Carbon monoxide is ubiquitous in the interstellar medium and is also predicted to be the dominant carbon-bearing gas in non-equilibrium models of the solar nebula [6]. As illustrated in Table 1, CO may also be the second-most abundant volatile in cometary nuclei. Prior to Halley, CO had been observed definitely in only two comets: the CO fourth positive group at $\lambda \sim 1500 \AA$ was detected during sounding rocket observations of comet West (1976 VI) [7] and during IUE observations of comet Bradfield (1979 X) [8]. The derived $\mathrm{CO}$ abundance in comet West was huge ( $\sim 30 \%$ of the $\mathrm{H}_{2} \mathrm{O}$ abundance). Similar observations were conducted on comet Halley [9], also showing a high $\mathrm{CO} / \mathrm{H}_{2} \mathrm{O}$ abundance ( $\sim 17 \%$ in this case). On the other hand, the UV observations of comet Bradfield yielded $\mathrm{CO} / \mathrm{H}_{2} \mathrm{O} \sim 0.02$ [10]. Does the relative abundance of $\mathrm{CO}$ vary from comet to comet? The Giotto NMS measurements of $\mathrm{CO}$ in the coma of comet Halley provided some interesting insight into this question [11]. The NMS observations showed that only $\sim 7 \%$ (or less) of the $\mathrm{CO}$ in the coma of Halley could be derived by sublimation from the nucleus. The rest of the $\mathrm{CO}$ in the coma is apparently produced from a distributed source, possibly due to the evaporation of organic mantles on cometary grains (the so-called CHON particles). Viewed from this new perspective, both the high $\mathrm{CO}$ abundances derived from UV observations of comets West and Halley, and the low $\mathrm{CO}$ abundance derived from the UV observation of comet Bradfield, are consistent. Whenever a large field-of-view (FOV) is used and the comet has a large dust production rate (the UV rocket observations of both West and Halley satisfied these conditions), a high abundance of $\mathrm{CO}$ will be observed in the coma due to extra CO production from grain evaporation. Whenever the FOV is small and/or the dust production rate is low, then the coma CO observation should be sampling something close to the "true" abundance in the nucleus (we are making a distinction here between the CO stored in the ice versus the presence of $\mathrm{C}-\mathrm{O}$ bonds in complex hydrocarbons that may be coating cometary grains).

Equilibrium condensation models of the solar nebula indicate that $\mathrm{CH}_{4}$ should be the dominant carbon-bearing gas in the outer regions of the nebula. Indeed, for many years people often referred to comets as being composed of "water, methane, and ammonia" 
although the latter two molecules had never been observed in comets and the indirect evidence for their presence indicated that they were only trace constituents. Recent nonequilibrium chemical models of the solar nebula demonstrate that $\mathrm{CH}_{4}$ should be extremely depleted relative to $\mathrm{CO}$ in the nebula, except near the sub-nebulae associated with the Giant planets [6].

Spectroscopic observations of the $\nu_{3}$ band of methane near $\lambda \sim 3.3 \mu \mathrm{m}$ have yielded the first direct evidence for the presence of $\mathrm{CH}_{4}$ in comets. The $\mathrm{R}(1)$ line of this band was marginally detected in comet Halley [12] while an average over six R-branch lines in this band gave a tentative detection during KAO observations of comet Wilson (1986l) [13]. The interpretation of these results in terms of the $\mathrm{CH}_{4}$ abundance is somewhat problematical. The derived abundance can vary significantly depending on the choice of the "effective" excitation temperature of the $\mathrm{CH}_{4}$ molecules in the coma, especially if only one line is used. Some of this uncertainty is reflected in the range of values given in Table 1 . We simply note that the above-referenced $\mathrm{KAO}$ observations of $\mathrm{H}_{2} \mathrm{O}$ indicate that the appropriate excitation temperature of $\mathrm{CH}_{4}$ is probably $\sim 50 \mathrm{~K}$ implying that the $\mathrm{CH}_{4}$ abundance is closer to $1 \%$ than $5 \%$.

Strong evidence for the presence of $\mathrm{CH}_{4}$ in the coma of comet Halley was also obtained by analysis of data taken by the ion mass spectrometer (IMS) on Giotto [21]. Using the $\mathrm{CH}_{3}^{+}$ion as a probe of the $\mathrm{CH}_{4}$ abundance in the coma, and by comparing the observed ion spectrum to a model spectrum containing all known important ion-molecule reactions, the IMS data indicate that $\mathrm{CH}_{4} / \mathrm{H}_{2} \mathrm{O} \sim 0.02$. The uncertainty in this number is difficult to establish owing to the complexity of the modeling, but the nominal value reported is consistent with the results from the spectroscopic investigations.

Both the NMS experiment on Giotto and the IR spectrometer on Vega detected $\mathrm{CO}_{2}$ in the coma of comet Halley and both measurements yield a $\mathrm{CO}_{2}$ relative abundance of $\sim 3 \%$ $[5,15]$. Assuming that the $\mathrm{CO}_{2}$ observed in these experiments originated in the nucleus, the $\mathrm{CO}_{2}$ abundance was approximately one-half of the $\mathrm{CO}$ abundance in Halley. UV observations of several comets indicate that the $\mathrm{CO}_{2} / \mathrm{H}_{2} \mathrm{O}$ abundance does not vary significantly from comet-to-comet (Feldman, private communication). If this is true, then $\mathrm{CO}_{2}$ and $\mathrm{CO}$ may have comparable abundances in some comets (e.g., comet Bradfeld [1979X]).

There was a tentative detection of $\mathrm{H}_{2} \mathrm{CO}$ in Halley via IR fluorescence in a vibrational band near $\lambda \sim 3.5 \mu \mathrm{m}$ by the IKS experiment [15]. The derived abundance from the IKS is given in Table 1 as an upper limit. If the detection stands, it indicates that $\mathrm{H}_{2} \mathrm{CO}$ is an important trace constituent in comets and may contribute significantly to the "extended" source of $\mathrm{CO}$ in the coma.

\subsection{MAJOR NITROGEN-BEARING SPECIES: $\mathrm{NH}_{3}, \mathrm{~N}_{2}, \mathrm{HCN}$}

There has only been one direct observation of $\mathrm{NH}_{3}$ in a comet: a radio line was marginally detected in comet IRAS-Araki-Alcock (1983d) [16]. The derivation of an $\mathrm{NH}_{3}$ abundance from these data is extremely model-dependent; the radio observers reported $\mathrm{NH}_{3} / \mathrm{H}_{2} \mathrm{O} \sim 0.06$ while I have derived a ratio of $\sim 0.003$ from the same data.

Since $\geq 90 \%$ of the photodissociation of $\mathrm{NH}_{3}$ by solar $\mathrm{UV}$ radiation results in the production of $\mathrm{NH}_{2}$, visible observations of the $\mathrm{NH}_{2}$ molecule should provide reasonably accurate measurements of the $\mathrm{NH}_{3}$ abundance. Observations of the $\mathrm{NH}_{2}$ band at $\lambda \sim 5700 \AA$ in 
comet Halley gave $\mathrm{NH}_{3} / \mathrm{H}_{2} \mathrm{O}=0.003$ [17].

Both $\mathrm{NH}_{3}$ and $\mathrm{CH}_{4}$ are required to explain the Giotto IMS data [14] (see the earlier discussion on $\mathrm{CH}_{4}$ ). An $\mathrm{NH}_{3}$ relative abundance of $\sim 1-2 \%$ gives the best fit of the model to the data. However, the accuracy of this result has not yet been determined due to the complexity of the modeling, and the uniqueness of the interpretation has also been challenged [18].

The abundance of $\mathrm{N}_{2}$ in comets is very difficult to establish with confidence. There are no known spectroscopic signatures of the neutral molecule and since its mass is coincident with that of $\mathrm{CO}$, even in situ observations with mass spectrometers suffer from a confusion. problem. Using the small difference in ionization potential between $\mathrm{N}_{2}$ and $\mathrm{CO}$, the Giotto NMS data were used to obtain $\mathrm{N}_{2} / \mathrm{H}_{2} \mathrm{O} \leq 0.1$ [11].

The $\mathrm{N}_{2}^{+}$ion is observed in the visible spectra of comets near $\lambda \sim 3914 \AA$. Since the ionization properties of $\mathrm{N}_{2}$ and $\mathrm{CO}$ are similar, and since the $\mathrm{CO} / \mathrm{H}_{2} \mathrm{O}$ abundance can be determined by other means, the ratio of the intensities of the visible bands of $\mathrm{N}_{2}$ and $\mathrm{CO}^{+}$ can be used to infer the ratio of $\mathrm{N} 2 / \mathrm{H}_{2} \mathrm{O}$. Using this technique for the Halley observations yields $\mathrm{N}_{2} / \mathrm{H}_{2} \mathrm{O} \leq 0.001$ (Feldman and Wyckoff, private communication).

Fluorescent emission from $\mathrm{CN}$ is usually the strongest feature in the visual spectra of comets. Most of the $\mathrm{CN}$ in the coma is probably produced by the photodissociation of HCN that has sublimated from the nucleus, although some may have been produced as a result of evaporation of organic-coated grains [19] or from photodissociation of more complex molecules [20]. The HCN molecule is detected via a rotational transition at radio frequencies, and the apparition of Halley witnessed the first systematic investigation of $\mathrm{HCN}$ in comets [21]. The derived abundance from these observations is $\mathrm{HCN} / \mathrm{H}_{2} \mathrm{O} \sim 0.001$.

\subsection{MAJOR SULFUR-BEARING SPECIES: $\mathrm{CS}_{2}, \mathrm{~S}_{\mathbf{2}}$}

Fluorescent emissions from CS and $S$ are a prominent features in UV spectra of comets [22]. The most plausible parent for $\mathrm{CS}$ is the $\mathrm{CS}_{2}$ molecule [23], although $\mathrm{CS}_{2}$ has never been observed directly. Assuming that all of the CS observed in the coma comes from $\mathrm{CS}_{2}$, then $\mathrm{CS}_{2} / \mathrm{H}_{2} \mathrm{O} \sim 0.001$, i.e., $\mathrm{CS}_{2}$ is a trace constituent of the nucleus.

UV observations of the "Earth-grazing" comet IRAS-Araki-Alcock (1983d) dramatically revealed the presence of $S_{2}$, a molecule which had never been observed previously in any astrophysical environment [24]. Since the $S_{2}$ discovery coincided with an "outburst" in cometary activity, and since the signal virtually disappeared during later observations, the presence of $\mathrm{S}_{2}$ in IRAS-Araki-Alcock may not be typical. The abundance derived from the UV observations during the outburst is $\mathrm{S}_{2} / \mathrm{H}_{2} \mathrm{O} \sim 0.001$.

The presence of the OCS molecule has been invoked to explain the spatial distribution of $S$ atoms in the coma [25]. Although this hypothesis is speculative since OCS has never been detected in comets, it is also clear that if OCS is present it cannot be more than a trace constituent of the nucleus.

\section{Conclusion}

Table 1 summarizes the abundance data on cometary volatiles discussed in this paper. All values are expressed as volume abundances relative to $\mathrm{H}_{2} \mathrm{O}$. Although the ability to com- 
pile a table like this with reasonable confidence represents a significant improvement in our knowledge of the nature of cometary matter, several cautionary remarks must be made. First, the abundances of trace species have been measured in very few comets. Table 1 is heavily weighted toward results from Halley since the best data generally come from this comet. Systematic observations of trace species in both "new" and "old" comets are desperately needed to establish whether the results from Halley, and the handful of other comets mentioned here, are "typical" of comets as a class. Second, even when identifications of species are secure, the conversion from raw data into nucleus abundances frequently introduces significant uncertainties (factors of two or more are common). Finally, comets often exhibit dramatic temporal variability which may suggest that the observed composition is not constant in time (e.g., the case of $S_{2}$ ). Despite these present shortcomings, we are well on our way toward a more quantitative understanding of the link between comet compositions and cometary formation environments.

\section{Acknowledgements}

I would like to express thanks to Bruce Fegley, Paul Feldman, Jonathan Lunine, Hal Larson, and Mike Mumma for many stimulating discussions on the topic of this paper.

TABLE 1. Volatile abundances

in comet nuclei

\begin{tabular}{ll}
\hline Molecule & Abundance \\
\hline $\mathrm{H}_{2} \mathrm{O}$ & 1 \\
$\mathrm{CO}$ & $0.02-0.07$ \\
$\mathrm{CH}_{4}$ & $0.01-0.05$ \\
$\mathrm{CO}_{2}$ & $\sim 0.03$ \\
$\mathrm{H}_{2} \mathrm{CO}$ & $\leq 0.05$ \\
$\mathrm{NH}_{3}$ & $0.003-0.02$ \\
$\mathrm{~N}_{2}$ & $\leq 0.001$ \\
$\mathrm{CS}_{2}$ & $\leq 0.001$ \\
$\mathrm{~S}_{2}$ & $\leq 0.001$ \\
$\mathrm{HCN}$ & $\sim 0.001$
\end{tabular}




\section{References}

1. Mumma, M.J., Weaver, H.A. , Larson, H.P., Davis, D.S. and Williams, M. (1986) 'Detection of water vapor in Halley's comet', Science, 232, 1523-1528.

2. Combes, M., et al. (1986) 'Infrared sounding of comet Halley from Vega 1', Nature, 321, 266-268.

3. Larson, H.P., Mumma, M.J., Weaver, H.A. and Davis, D.S. (1986) 'Velocity-Resolved observations of water in comet Halley', Ap. J. Lett., 309, L95-L99.

4. Weaver, H.A., Mumma, M.J., Larson, H.P. and Davis, D.S. (1986) 'Post-Perihelion observations of water in comet Halley', Nature, 324, 441-444.

5. Krankowsky, D., et al. (1986) 'In situ gas and ion measurements at comet Halley', Nature, 320, 326-329.

6. Prinn, R.G. and Fegley, B. (1988) 'Solar nebula chemistry: origin of planetary, satellite, and cometary volatiles', in S. Atreya, J. Pollack, and M. Matthews (eds.), Origin and Evolution of Planetary and Satellite Atmospheres, University of Arizona Press, in press.

7. Feldman, P.D. and Brune, W.H. (1977) 'Carbon production in comet West 1975n', Ap. J. Lett., 209, L45-L48.

8. Feldman, P.D., et al. (1981) 'IUE Observations of the UV spectrum of comet Bradfield, Nature, 286, 132-135.

9. Woods, T.N., Feldman, P.D., Dymond, K.F. and Sahnow, D.J. (1986) 'Rocket ultraviolet spectroscopy of comet Halley and abundance of carbon monoxide and carbon', Nature, 324, 436-438.

10. A'Hearn, M.F. and Feldman, P.D. (1980) 'Carbon in comet Bradfield (1979l)', Ap. J. Lett., 242, L187-L190.

11. Eberhardt, P., et al. (1987) 'The $\mathrm{CO}$ and $\mathrm{N}_{2}$ abundance in comet p/Halley', Astron. Ap., 187, 481-484.

12. Kawara, K., Gregory, B., Yamamoto, T. and Shibai, H. (1988) 'Infrared spectroscopic observation of methane in comet Halley', Astron. Ap., in press.

13. Larson, H.P., Weaver, H.A., Mumma, M.J. and Drapatz, S. (1988) 'Airborne infrared spectroscopy of comet Wilson (1986I) and comparisons with comet Halley', Ap. J., in press.

14. Allen, M., et al. (1987) 'Evidence for methane and ammonia in the coma of comet p/Halley', Astron. Ap., 187, 502-512.

15. Moroz, V.I., et al. (1987) 'Detection of parent molecules in comet $\mathrm{p} / \mathrm{Halley}$ from the IKS-Vega experiment', Astron. Ap., 187, 513-518. 
16. Altenhoff, W.J., et al. (1983) 'Radio observations of comet 1983d', Astron. Ap., 125, L19-L22.

17. Wyckoff, S., Tegler, S., Wehinger, P., Spinrad, H. and Belton, M.J.S. (1988) 'Abundances in comet Halley at the time of the spacecraft encounters', Ap. J., 325, 927-938.

18. Marconi, M.L. and Mendis, D.A. (1988) 'On the ammonia abundance in the coma of Halley's comet', Ap. J., 330, 513-517.

19. A'Hearn, M.F., et al. (1986) 'Cyanogen jets in comet Halley', Nature, 324, 649-651.

20. Bockelée-Morvan, D. and Crovisier, J. (1986) 'Possible parents for the cometary CN radical: photochemistry and excitation conditions', Astron. Ap., 151, 90-100.

21. Schloerb, F.P., Kinzel, W.M., Swade, D.A. and Irvine, W.M. (1987) 'Observations of HCN in comet p/Halley', Astron. Ap., 187, 475-480.

22. Weaver, H.A., et al. (1982) 'IUE observations of faint comets', Icarus, 47, 449-463.

23. Jackson, W.M., Halpern, J.B., Feldman, P.D. and Rahe, J. (1982) 'Production of CS and $S$ in comet Bradfield (1979X)', Astron. Ap., 107, 385-389.

24. A'Hearn, M.F., Feldman, P.D., and Schleicher, D.G. (1983) 'The Discovery of $S_{2}$ in comet IRAS-Araki-Alcock 1983d', Ap. J. Lett., 274, L99-103.

25. Azoulay, G. and Festou, M.C. (1985) 'The abundance of sulfur in comets', in C.-I. Lagerkvist, B.A. Lindblad, H. Lundstedt and G. Rickman (eds.), Comets, Asteroids, and Meteors II, pp. 273-276. 